\title{
INVENTORYING AND MONITORING OF ALPINE SPECIFIC GENERA AND SPECIES OF HARAMOSH AND BAGRAOTE VALLEYS., (KARAKORAM RANGE) GILGIT-BALTISTAN, PAKISTAN
}

\author{
Sher Wali Khan ${ }^{1,6^{*}, \text { Surayya Khatoon }}$, Azhar Hussain ${ }^{3}$, Haider Abbas ${ }^{4,7^{*}}$ \\ and Haibat $\mathrm{Ali}^{5}$ \\ ${ }^{1}$ Department of Biological Sciences, Karakoram International University, Gilgit-Pakistan; ${ }^{2}$ Department of Botany, \\ University of Karachi, Karachi-Pakistan; ${ }^{3}$ Department of Agriculture and Food technology, Karakoram \\ International University, Gilgit-Pakistan; ${ }^{4}$ Department of Agriculture and Agribusiness Management, University of \\ Karachi, Karachi-Pakistan; ${ }^{5}$ Current Address: AgCall Inc./Dow AgroSciences Inc. Saskatoon, SK. \\ Canada, ${ }^{6}$ Department of Environmental Sciences, Karakoram International University, Gilgit-Pakistan. \\ "Corresponding author's e-mail: dr.sherwali@ kiu.edu.pk
}

\begin{abstract}
Inventorying and monitoring work on alpine specific flora of Haramosh and Bagrote valleys (Gilgit-Baltistan) was done for fourteen years from 2001-2014. The fourteen years inventorying revealed a rich alpine exclusive species consisting of 105 species in 52 genera and 21 families of Angiosperms. The Alpine zone had 13 genera with 3 or more alpine exclusive species; Potentilla and Carex with 9 species each were the largest genera of this zone, followed by and Draba (8 species) Rhodiola 5 species etc. Genera containing 9 or 10 species occurred only in Alpine zone which are specific to extreme alpine habitat. The exclusive Alpine flora of both valleys formed $45.25 \%$ of the total alpine flora. Although the total number of species was the highest in the Sub-alpine zone, but in the species specific to any one zone, the Alpine zone had the highest number, that is, 105 of the total 232 species were exclusively found in this zone only. Like species, 22 genera out of 52 were exclusively found in alpine zone only. The Alpine zone was characterized by herbs and low shrubs, with Potentilla species as the dominants. A clear trend of migration of certain species both from lower to higher latitudes and altitudes was observed. Bistorta affinis and Potentilla species were observed that are grazing resistant and drought resistant species of the alpine zone were upward shifted from subalpine zone in both valleys during the last fourteen years. Beside these dominant and grazing resistant species some other species like Saussurea simpsoniana, Corydalis spp., Pulsatilla wallichiana, Anemone rupicola, Allium spp., Silene gonosperma, Aconogonon spp., Primula spp., were upward shifted within the alpine zone and some are completely shifted from subalpine zone. Anemone rupicola, Pulsatilla wallichina and Aconogonon spp. were completely shifted from lower zone to alpine zone of both valleys. The species richness index of Alpine zone however showed increasing trend probably due to species migrations towards the alpine zone.
\end{abstract}

Keywords: Karakoram Range, alpine species, floristic survey, biodiversity, ecosystems.

\section{INTRODUCTION}

Both valleys are the part of the Karakoram range that lying in the north-eastern side of the capital city Gilgit (Fig. 1) between $35.50-36.5^{\circ} \mathrm{N}$ latitude and $74.54^{\circ} \mathrm{E}$ longitude, covering an area of 2340 sq.km (Khan and Khatoon, 2007). The alpine zone has a fragmental and highly scattered distribution over the mountain system of the Karakoram Range. The large alpine areas are concentrated in the upper villages of both valleys with more than 60 mountain peaks acceding $4000 \mathrm{~m}$. The area is surrounded by high mountains of the Karakoram, Himalayas and Hindukush Ranges (Perkin, 2003). Both valleys are the part of the Eastern Irano-Turanian sub-region. This sub-region is confined to the northern mountainous region of Pakistan and Kashmir between $35^{\circ}$ $36^{\circ} \mathrm{NL}$ (Ali and Qaiser, 1986). The alpine zone includes the upper most reaches of the entire region from $3500 \mathrm{~m}$ to permanent snow line. The word "Alpine" is normally used to denote a mountainous region above the tree-line or timberline, lacking tree habitation (Noroozi et al., 2008), but abounding in low herbs and a few shrubs. Inventorying of biodiversity is the baseline study for the exploration of the earth surface and conservation, sustainable use, and management of the biodiversity elements and monitor changes over the passage of time, (Stork and Samways, 1995). Thus, baseline inventorying and monitoring information is a necessary first step in conservation of biodiversity elements. During an inventory of a region the collected voucher specimens are crucial to obtain accurate identification of plants present in a study area (Dugan et al., 2007). All living beings obtain their life support material from their environment. The twentieth century was the warmest century, and 1990 to 2000 was the warmest decade, of the past millennium (Hardy, 2003) and warming trends have been 
observed in the elevated regions such as the HimalayanTibetan region, leading to the retreat of glaciers (Ramanathan, 2007). During just the last 150 years, the earth's global average temperature has increased by about $0.8^{\circ} \mathrm{C}$ and at higher latitudes has increased by several degrees Celsius (Dodd, 1994). In fact, the last decade of the twentieth century was the warmest in the entire global instrumental record. Changes in species diversity occur naturally overtime in all communities and ecosystems. Species based monitoring documents to establish a baseline data for understanding the impact of natural disturbance on species composition and abundance in ecosystem (Watson and Novelly, 2004). The floristic inventories play a significant role in increasing our understanding and information level on availability of resources and its relationship with the mankind. This study aims at finding out the exclusive alpine plant species and genera of the both valleys of district Gilgit and enumerates the dominant alpine specific genera and species in alpine zones of the area. The documentation of alpine species and characterization of their altitudinal ranges is based on Flora of Pakistan and our own field surveys.

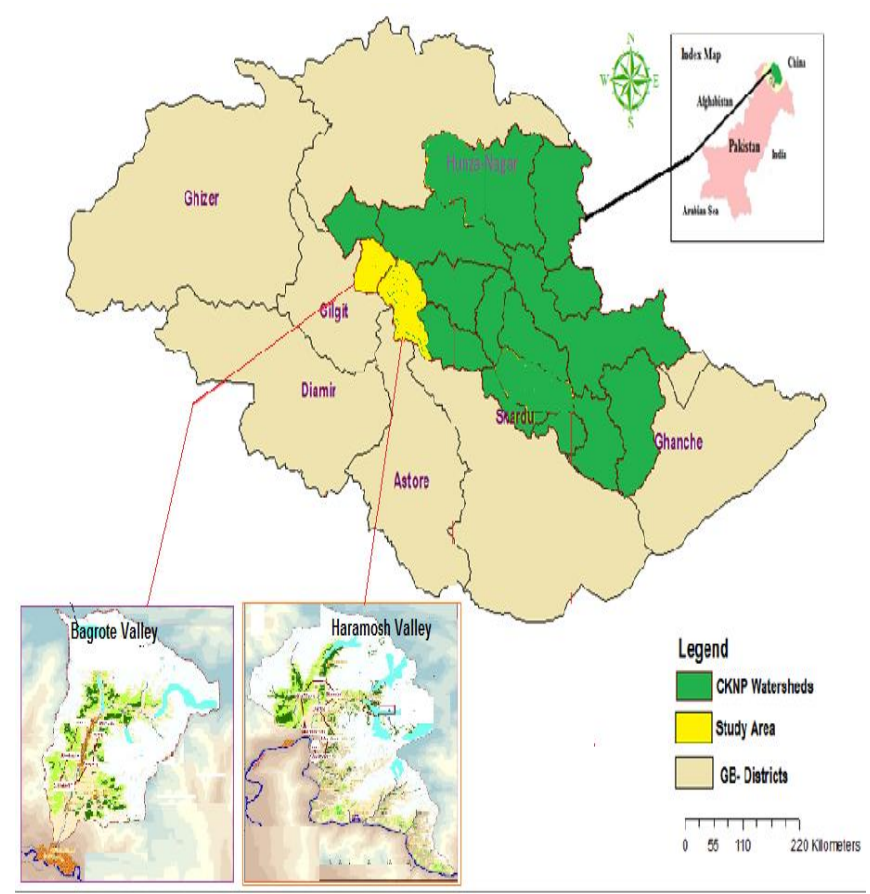

Figure1. Map of the study area.

\section{MATERIALS AND METHODS}

The present study was mainly focused on the extreme alpine ecosystem and habitats of Haramosh and Bugrote valleys of Gilgit-Baltistan, Pakistan. An intensive floristic survey was carried out during the summer months i.e. from April to September 2001-2014, for the collection and identification of plants in various habitats between the altitudinal ranges of
$3500-4800 \mathrm{~m}$. The area surveyed including various alpine pastures, stream and spring banks, late lying snow patch areas and moist alpine rocky places etc. Extensive field information was collected based on habit, habitat, altitude and abundance. To collect the maximum information on aspects of flora, field notes regarding the plant distribution pattern, composition, altitudinal aspect and topographic condition were noted down. For the study of herbaceous communities and their altitudinal distribution of different species, the altitude of every species was recorded with the help of GPS. The collected plant specimens from various localities of both valleys were identified with the help of Flora of Pakistan (Nasir and Ali, 1970-89; Ali and Nasir, 1989-1991; Ali and Qaiser, 1993-2012), Stewart (1972), some other relevant Floras of the neighboring countries, and also by comparing with the authentic specimens available in the Karachi University Herbarium. After identification voucher specimens have been housed in the Karachi University Herbarium (KUH) and newly established Karakoram International University Herbarium. During the first two years, i.e. 2001 and 2002, an inventory of plant species was prepared on the basis of thorough collection of specimens in the study area; to serve as the base-line for monitoring. Monitoring was done during 2003 to 2007 through random sampling by quadrat method. Twenty quadrats from the selected sites were taken each year in alpine zone. Squareshaped quadrats of $1 \times 1 \mathrm{~m}$ were taken in the selected sites of Alpine zones. The number of individuals of each species in each quadrat was counted and their basal area measured. From above information the following attributes were calculated: relative frequency (RF), relative density (RD), relative cover (RC) and importance value index (IVI) = $\mathrm{RC}+\mathrm{RD}+\mathrm{RF}$. Life- form categories of Raunkiaer's system (Raunkiaer, 1934), as presented by Ellenberg et al. (1991) were accepted.

\section{RESULTS}

A total of 103 exclusively alpine species including 22 monocot and 81 dicot species were identified from alpine zone of both valleys representing 52 genera and 21 families of Angiosperms (Table 1). The habits of these 103 plant species comprised 2 annuals, 2 small shrubs and 99 perennial herbs. The alpine specific species diversity comprised $45.25 \%$ of alpine flora and $18.75 \%$ of the entire flora 560 species reported from both valleys. High altitude regions are characterized by cold and arid climate and landscapes of many microhabitats, exhibiting a vegetation of specific adaptation results in a peculiar life form of alpine species (Kumar and Sharma, 2013). Both valleys with temperate and alpine climate provide a suitable habitat for the growth of alpine plant species. The dominant families with more than 5 exclusive alpine species in both valleys of Karakoram range were Asteraceae with 16 species) followed by Ranunculaceae 
(with 11 species), Brassicaceae, Rosaceae and Cyperaceae Crassullaceae and Apiaceae (with 5 species each) whereas the (with 10 species each), Scrophulariaceae (7 species), dominant exclusive alpine genera were Potentilla and Carex Table 1. Distribution of Alpine specific plants, their, life form, habit, altitude and palatability.

\begin{tabular}{|c|c|c|c|c|c|c|c|c|}
\hline S\# & Family & Name of species & Habit & Life form & Altitude & Remarks & Medicinal value & Palatability \\
\hline 1 & Alliaceae & Allium carolinianum DC. & $\mathrm{P}$ & G & $3600 \mathrm{~m}$ & $\mathrm{C}$ & Low medicinal value & LP \\
\hline 2 & Alliaceae & Allium oreoprasum Schrenk & $\mathrm{P}$ & $\mathrm{G}$ & $3700 \mathrm{~m}$ & Inf. & Not known & LP \\
\hline 3 & Apiaceae & Pleurospermum candollei (DC.) C.B.Clarke. & $\mathrm{P}$ & $\mathrm{H}$ & $4000 \mathrm{~m}$ & $\mathrm{C}$ & High medicinal value & HP \\
\hline 4 & Apiaceae & Pleurospermum hookeri var. thomsonii Clarke & $\mathrm{P}$ & $\mathrm{H}$ & $4000 \mathrm{~m}$ & $\mathrm{C}$ & Low medicinal value & HP \\
\hline 5 & Apiaceae & $\begin{array}{l}\text { Pleurospermum stellatum Benth. var. lindleyanum (K1.) } \\
\text { C.B.Clarke }\end{array}$ & $\mathrm{P}$ & $\mathrm{H}$ & $4000 \mathrm{~m}$ & $\mathrm{C}$ & Not known & $\mathrm{HP}$ \\
\hline 6 & Apiaceae & Pleurospermum stylosum Clarke & $\mathrm{P}$ & $\mathrm{H}$ & $3600 \mathrm{~m}$ & $\mathrm{C}$ & Not known & HP \\
\hline 7 & Apiaceae & Vicatia coniifolia DC. & $\mathrm{P}$ & $\mathrm{H}$ & $4000 \mathrm{~m}$ & $\mathrm{C}$ & Not known & LP \\
\hline 8 & Asteraceae & Achillea millefolium $\mathrm{L}$ ssp. Millefolium & $\mathrm{P}$ & $\mathrm{H}$ & $3700 \mathrm{~m}$ & Inf. & Low medicinal value & NP \\
\hline 9 & Asteraceae & Allardia glabra Decne. & $\mathrm{P}$ & $\mathrm{H}$ & $3900 \mathrm{~m}$ & $\mathrm{C}$ & Not known & NP \\
\hline 10 & Asteraceae & Allardia nivea Hook.f.\& Thomson ex C.B. Clarke & $\mathrm{P}$ & $\mathrm{Ch}$ & $4100 \mathrm{~m}$ & Inf. & Not known & NP \\
\hline 11 & Asteraceae & Allardia stoliczkae C.B.Clarke & $\mathrm{P}$ & $\mathrm{H}$ & $4200 \mathrm{~m}$ & Inf. & Not known & NP \\
\hline 12 & Asteraceae & Allardia tridactylites (Kar.\& Kir.) Schultz-Bip. & $\mathrm{P}$ & $\mathrm{Ch}$ & $4200 \mathrm{~m}$ & Inf. & Not known & NP \\
\hline 13 & Asteraceae & Aster peduncularis Wall.ex Nees & $\mathrm{P}$ & $\mathrm{H}$ & $4000 \mathrm{~m}$ & $\mathrm{C}$ & Not known & LP \\
\hline 14 & Asteraceae & Cicerbita sp. nov. & $\mathrm{P}$ & $\mathrm{H}$ & $4000 \mathrm{~m}$ & $\mathrm{C}$ (endemic) & Not known & LP \\
\hline 15 & Asteraceae & Cremanthodium decaisnei Clarke & $\mathrm{P}$ & $\mathrm{H}$ & $4400 \mathrm{~m}$ & $\mathrm{C}$ & Not known & LP \\
\hline 16 & Asteraceae & Doronicum falconeri Clarke & $\mathrm{P}$ & $\mathrm{H}$ & $3800 \mathrm{~m}$ & RR & Not known & LP \\
\hline 17 & Asteraceae & Erigeron alpinum $\mathrm{L}$. & $\mathrm{P}$ & $\mathrm{H}$ & $3600 \mathrm{~m}$ & $\mathrm{C}$ & Not known & LP \\
\hline 18 & Asteraceae & Hippolytia.dolichophylla (Kitam.) Bremer \& Humphries & $\mathrm{P}$ & $\mathrm{H}$ & $3600 \mathrm{~m}$ & Inf. & Not known & LP \\
\hline 19 & Asteraceae & Saussurea falconeri Hook.f. & $\mathrm{P}$ & $\mathrm{H}$ & $4000 \mathrm{~m}$ & Inf. & Highly medicinal value & LP \\
\hline 20 & Asteraceae & Saussurea jacea (Klotz.) Clarke & $\mathrm{P}$ & $\mathrm{H}$ & $3900 \mathrm{~m}$ & Inf. & High medicinal value & LP \\
\hline 21 & Asteraceae & Saussurea simpsoniana (Field \& Gardner) Lipschitz. & $\mathrm{P}$ & $\mathrm{H}$ & $4800 \mathrm{~m}$ & Inf. & highly medicinal value & NP \\
\hline 22 & Asteraceae & Senecio graciliflorus DC. & $\mathrm{P}$ & $\mathrm{H}$ & $4000 \mathrm{~m}$ & Inf. & Not known & LP \\
\hline 23 & Asteraceae & Tanacetum falconeri Hook.f. & $\mathrm{P}$ & $\mathrm{H}$ & $3600 \mathrm{~m}$ & $\mathrm{C}$ & Highly medicinal value & LP \\
\hline 24 & Brassicaceae & $\begin{array}{l}\text { Arabidopsis mollissima } \\
\text { (C.A Mey.) N.Busch }\end{array}$ & $\mathrm{P}$ & $\mathrm{H}$ & $4000 \mathrm{~m}$ & $\mathrm{C}$ & Not known & LP \\
\hline 25 & Brassicaceae & Draba altaica (C.A.Mey.)Bunge & $\mathrm{P}$ & $\mathrm{H}$ & $4200 \mathrm{~m}$ & $\mathrm{C}$ & Not known & LP \\
\hline 26 & Brassicaceae & Draba cachemirica Gandoger & $\mathrm{P}$ & $\mathrm{H}$ & $4000 \mathrm{~m}$ & $\mathrm{C}$ & Not known & LP \\
\hline 27 & Brassicaceae & Draba lanceolata Royle & $\mathrm{P}$ & $\mathrm{H}$ & $4000 \mathrm{~m}$ & Inf. & Not known & LP \\
\hline 28 & Brassicaceae & Draba melanopus Komarov & $\mathrm{P}$ & $\mathrm{H}$ & $4000 \mathrm{~m}$ & $\mathrm{C}$ & Not known & LP \\
\hline 29 & Brassicaceae & Draba oreades Schrenk & $\mathrm{P}$ & $\mathrm{H}$ & $4200 \mathrm{~m}$ & $\mathrm{C}$ & Not known & LP \\
\hline 30 & Brassicaceae & Draba setosa Royle & $\mathrm{P}$ & $\mathrm{H}$ & $4200 \mathrm{~m}$ & $\mathrm{C}$ & Not known & LP \\
\hline 31 & Brassicaceae & Draba stenocarpa Hook.f. \& Thoms. & $\mathrm{P}$ & $\mathrm{H}$ & $4200 \mathrm{~m}$ & $\mathrm{C}$ & Not known & LP \\
\hline 32 & Brassicaceae & $\begin{array}{l}\text { Draba tibetica Hook.f. \& Thoms. var. chitralensis } \\
\text { (Schultz) Jafri }\end{array}$ & $\mathrm{P}$ & $\mathrm{H}$ & $4000 \mathrm{~m}$ & $\mathrm{C}$ & Not known & LP \\
\hline 33 & Brassicaceae & Thlaspi andersonii (Hook. f. \& Thom.) O. E. Schulz+ & $\mathrm{P}$ & $\mathrm{H}$ & $4000 \mathrm{~m}$ & $\mathrm{R}$ & Not known & LP \\
\hline 34 & Campanulaceae & Adenophora himalayana Feer & $\mathrm{P}$ & $\mathrm{H}$ & $3700 \mathrm{~m}$ & $\mathrm{C}$ & Not known & NP \\
\hline 35 & Caryophyllaceae & $\begin{array}{l}\text { Silene gonosperma (Rupr.) Bocquet ssp. himalayensis } \\
\text { (Rohrb.) Bocquet }\end{array}$ & $\mathrm{P}$ & $\mathrm{H}$ & $4000 \mathrm{~m}$ & Inf. & Not known & $\mathrm{NP}$ \\
\hline 36 & Crassulaceae & Rhodiola heterodonta (Hook. f. \& Thom.) Boriss. & $\mathrm{P}$ & $\mathrm{H}$ & $4000 \mathrm{~m}$ & $\mathrm{C}$ & Not known & NP \\
\hline 37 & Crassulaceae & Rhodiola quadrifida (Pallas) Schrenk & $\mathrm{P}$ & $\mathrm{H}$ & $4000 \mathrm{~m}$ & $\mathrm{C}$ & Not known & NP \\
\hline 38 & Crassulaceae & Rhodiola recticaulis Boriss. & $\mathrm{P}$ & $\mathrm{H}$ & $4000 \mathrm{~m}$ & $\mathrm{C}$ & Not known & NP \\
\hline 39 & Crassulaceae & Rhodiola tibetica (Hook.f. \& Thom.) S.H.Fu & $\mathrm{P}$ & $\mathrm{H}$ & $3800 \mathrm{~m}$ & $\mathrm{C}$ & Not known & NP \\
\hline 40 & Crassulaceae & Rhodiola wallichiana (Hook.f.) S.H.Fu & $\mathrm{P}$ & $\mathrm{H}$ & $3700 \mathrm{~m}$ & $\mathrm{C}$ & Not known & NP \\
\hline 41 & Cyperaceae & Carex canescens $\mathrm{L}$. & $\mathrm{P}$ & $\mathrm{H}$ & $4000 \mathrm{~m}$ & $\mathrm{CC}$ & Not known & HP \\
\hline 42 & Cyperaceae & Carex cardiolepis Nees & $\mathrm{P}$ & $\mathrm{H}$ & $4000 \mathrm{~m}$ & $\mathrm{C}$ & Not known & HP \\
\hline 43 & Cyperaceae & Carex cruenta Nees & $\mathrm{P}$ & $\mathrm{H}$ & $4300 \mathrm{~m}$ & $\mathrm{C}$ & Not known & HP \\
\hline 44 & Cyperaceae & Carex divisa Huds. & $\mathrm{P}$ & $\mathrm{H}$ & $4000 \mathrm{~m}$ & $\mathrm{C}$ & Not known & $\mathrm{HP}$ \\
\hline 45 & Cyperaceae & Carex melanantha C. A. Mey. & $\mathrm{P}$ & $\mathrm{H}$ & $3800 \mathrm{~m}$ & $\mathrm{C}$ & Not known & HP \\
\hline 46 & Cyperaceae & Carex nivalis Boott & $\mathrm{P}$ & $\mathrm{H}$ & $4100 \mathrm{~m}$ & $\mathrm{C}$ & Not known & $\mathrm{HP}$ \\
\hline 47 & Cyperaceae & Carex obscura Nees & $\mathrm{P}$ & $\mathrm{H}$ & $4300 \mathrm{~m}$ & $\mathrm{C}$ & Not known & $\mathrm{HP}$ \\
\hline 48 & Cyperaceae & Carex oligocarya C.B.Clarke & $\mathrm{P}$ & $\mathrm{H}$ & $4000 \mathrm{~m}$ & $\mathrm{C}$ & Not known & $\mathrm{HP}$ \\
\hline 49 & Cyperaceae & Carex pseudofoetida ssp. afghanica Kukkonen & $\mathrm{P}$ & $\mathrm{H}$ & $4100 \mathrm{~m}$ & $\mathrm{C}$ & Not known & $\mathrm{HP}$ \\
\hline 50 & Cyperaceae & Eleocharis quinqueflora (F.X.Hartm.) O.Schwarz & $\mathrm{P}$ & $\mathrm{H}$ & $3800 \mathrm{~m}$ & $\mathrm{CC}$ & Not known & $\mathrm{HP}$ \\
\hline 51 & Ericaceae & Rhododendron hypenanthum Balf. f. & Sh & $\mathrm{Ph}$ & $3600 \mathrm{~m}$ & $\mathrm{C}$ & Low medicinal value & NP \\
\hline 52 & Fumariaceae & Corydalis falconeri Hook.f. \& Thoms. & $\mathrm{P}$ & $\mathrm{Ch}$ & $4000 \mathrm{~m}$ & $\mathrm{C}$ & Low medicinal value & LP \\
\hline 53 & Fumariaceae & Corydalis gortschakovii Schrenk & $\mathrm{P}$ & $\mathrm{Ch}$ & $4000 \mathrm{~m}$ & $\mathrm{C}$ & Low medicinal value & LP \\
\hline 54 & Liliaceae & Gagea lowariensis Pascher & $\mathrm{P}$ & $\mathrm{G}$ & $3800 \mathrm{~m}$ & $\mathrm{C}$ & Not known & LP \\
\hline 55 & Liliaceae & Gagea spumosa Levichev & $\mathrm{P}$ & G & $4200 \mathrm{~m}$ & $\mathrm{C}$ & Not known & LP \\
\hline 56 & Liliaceae & Lloydia serotina $(\mathrm{L}$.$) Rchb.$ & $\mathrm{P}$ & $\mathrm{G}$ & $4000 \mathrm{~m}$ & $\mathrm{C}$ & Not known & LP \\
\hline 57 & Papaveraceae & Papaver nudicaule $\mathrm{L}$. & $\mathrm{A}$ & Th & $4000 \mathrm{~m}$ & $\mathrm{C}$ & Not known & LP \\
\hline 58 & Poaceae & Agrostis gigantea Roth & $\mathrm{P}$ & $\mathrm{Ch}$ & $3650 \mathrm{~m}$ & $\mathrm{C}$ & Not known & $\mathrm{HP}$ \\
\hline 59 & Poaceae & Alopecurus himalaicus Hook.f. & $\mathrm{P}$ & $\mathrm{H}$ & $4000 \mathrm{~m}$ & Inf. & Not known & HP \\
\hline 60 & Poaceae & Festuca alaica Drobov & $\mathrm{P}$ & $\mathrm{H}$ & $4000 \mathrm{~m}$ & $\mathrm{C}$ & Not known & $\mathrm{HP}$ \\
\hline 61 & Poaceae & Festuca alatavica (St.-Yves) Rozhev. & $\mathrm{P}$ & $\mathrm{H}$ & $4000 \mathrm{~m}$ & $\mathrm{C}$ & Not known & $\mathrm{HP}$ \\
\hline 62 & Poaceae & Phleum alpinum $\mathrm{L}$. & $\mathrm{P}$ & $\mathrm{H}$ & $4000 \mathrm{~m}$ & $\mathrm{C}$ & Not known & $\mathrm{HP}$ \\
\hline 63 & Poaceae & Poa alpina $\mathrm{L}$. & $\mathrm{P}$ & $\mathrm{H}$ & $4000 \mathrm{~m}$ & $\mathrm{C}$ & Not known & $\mathrm{HP}$ \\
\hline 64 & Polygonaceae & Aconogonon coriarium (Grig.) Sojak. & $\mathrm{P}$ & $\mathrm{Ch}$ & $4000 \mathrm{~m}$ & $\mathrm{C}$ & Not known & LP \\
\hline 65 & Polygonaceae & $\begin{array}{l}\text { Aconogonon tortuosum (D.Don) Hara var. tibetanum } \\
\text { (Meisn.)S.-P.Hong }\end{array}$ & $\mathrm{P}$ & $\mathrm{Ch}$ & $4000 \mathrm{~m}$ & Inf. & Not known & LP \\
\hline 66 & Polygonaceae & Aconogonon tortuosum (D.Don) Hara var. tortuosum & $\mathrm{P}$ & $\mathrm{H}$ & $4000 \mathrm{~m}$ & Inf. & Not known & LP \\
\hline 67 & Primulaceae & Cortusa brotheri Pax ex Lipsky & A & Th & $3600 \mathrm{~m}$ & $\mathrm{C}$ & Not known & LP \\
\hline
\end{tabular}


Khan, Khatoon, Hussain, Abbas \& Ali

\begin{tabular}{|c|c|c|c|c|c|c|c|c|}
\hline S\# & Family & Name of species & Habit & Life form & Altitude & Remarks & Medicinal value & Palatability \\
\hline 68 & Primulaceae & Primula reptans Hook.ex Watt & $\mathrm{P}$ & $\mathrm{H}$ & $4200 \mathrm{~m}$ & $\mathrm{C}$ & Not known & NP \\
\hline 69 & Ranunculaceae & $\begin{array}{l}\text { Aconitum violaceum Jacq. ex Stapf var. weileri (Gilli) } \\
\text { H.Riedle }\end{array}$ & $\mathrm{P}$ & G & $3700 \mathrm{~m}$ & Inf. (endemic) & High medicinal value & LP \\
\hline 70 & Ranunculaceae & Aconitum violaceum Jacq.ex Stapf var. violaceum & $P$ & $\mathrm{G}$ & $3600 \mathrm{~m}$ & Inf. & High medicinal value & LP \\
\hline 71 & Ranunculaceae & $\begin{array}{l}\text { Aquilegia moorcroftiana Wall.ex Royle var. } \\
\text { moorcroftiana }\end{array}$ & $\mathrm{P}$ & $\mathrm{H}$ & $3600 \mathrm{~m}$ & $\mathrm{C}$ & Not known & LP \\
\hline 72 & Ranunculaceae & Anemone rupicola $\mathrm{Camb}$. & $\mathrm{P}$ & $\mathrm{H}$ & $3650 \mathrm{~m}$ & $\mathrm{C}$ & Not known & LP \\
\hline 73 & Ranunculaceae & $\begin{array}{l}\text { Callianthemum pimpinelloides (D.Don ex Royle) Hook.f. } \\
\text { \& Thoms. }\end{array}$ & $\mathrm{P}$ & $\mathrm{H}$ & $4200 \mathrm{~m}$ & $\mathrm{C}$ & Not known & LP \\
\hline 74 & Ranunculaceae & Delphinium brunonianum Royle & $\mathrm{P}$ & $\mathrm{H}$ & $4000 \mathrm{~m}$ & $\mathrm{C}$ & High medicinal value & LP \\
\hline 75 & Ranunculaceae & Delphinium pyramidale Royle & $\mathrm{P}$ & $\mathrm{Ch}$ & $4200 \mathrm{~m}$ & $\mathrm{C}$ & High medicinal value & LP \\
\hline 76 & Ranunculaceae & Delphinium vestitum Wall.ex Royle & $\mathrm{P}$ & $\mathrm{H}$ & $4300 \mathrm{~m}$ & Inf. & High medicinal value & LP \\
\hline 77 & Ranunculaceae & Isopyrum anemonoides Kar. \& Kir. & $\mathrm{P}$ & $\mathrm{H}$ & $4000 \mathrm{~m}$ & $\mathrm{C}$ & Not known & NP \\
\hline 78 & Ranunculaceae & Paraquilegia anemonoides (Willd.) Ulbr. & $P$ & $\mathrm{H}$ & $3600 \mathrm{~m}$ & Inf. & Not known & NP \\
\hline 79 & Ranunculaceae & Ranunculus stewartii H.Riedl & $P$ & $\mathrm{H}$ & $4000 \mathrm{~m}$ & Inf. & Not known & NP \\
\hline 80 & Rosaceae & Cotoneaster uniflora Bunge & $\mathrm{Sh}$ & $\mathrm{Ph}$ & $3600 \mathrm{~m}$ & $\mathrm{R}$ & Not known & LP \\
\hline 81 & Rosaceae & Potentilla anserina $\mathrm{L}$. & $P$ & $\mathrm{H}$ & $3700 \mathrm{~m}$ & $\mathrm{CC}$ & Not known & LP \\
\hline 82 & Rosaceae & Potentilla argyrophylla Wall.ex Lehm. & $\mathrm{P}$ & $\mathrm{H}$ & $3600 \mathrm{~m}$ & $\mathrm{C}$ & Not known & $\mathrm{HP}$ \\
\hline 83 & Rosaceae & Potentilla atrosanguinea Lodd. & $P$ & $\mathrm{H}$ & $3600 \mathrm{~m}$ & $\mathrm{C}$ & Not known & $\mathrm{HP}$ \\
\hline 84 & Rosaceae & Potentilla doubjouneana Camb. & $\mathrm{P}$ & $\mathrm{H}$ & $3700 \mathrm{~m}$ & $\mathrm{C}$ & Not known & $\mathrm{HP}$ \\
\hline 85 & Rosaceae & Potentilla eriocarpa Wall.ex Lehm. & $\mathrm{P}$ & $\mathrm{H}$ & $3600 \mathrm{~m}$ & $\mathrm{C}$ & Not known & HP \\
\hline 86 & Rosaceae & Potentilla gelida C.A.Mey. & $P$ & $\mathrm{H}$ & $3600 \mathrm{~m}$ & $\mathrm{C}$ & Not known & $\mathrm{HP}$ \\
\hline 87 & Rosaceae & Potentilla gerardiana Lindl.ex Lehm. & $\mathrm{P}$ & $\mathrm{H}$ & $3700 \mathrm{~m}$ & $\mathrm{C}$ & Not known & $\mathrm{HP}$ \\
\hline 88 & Rosaceae & Potentilla multifida L. & $\mathrm{P}$ & $\mathrm{H}$ & $3600 \mathrm{~m}$ & $\mathrm{C}$ & Not known & HP \\
\hline 89 & Rosaceae & Potentilla turezainowiana Stesh. & $\mathrm{P}$ & $\mathrm{H}$ & $3700 \mathrm{~m}$ & Inf. & Not known & $\mathrm{HP}$ \\
\hline 90 & Saxifragaceae & $\begin{array}{l}\text { Saxifraga flagellaris Willd.ex Sternb ssp. stenophylla } \\
\text { (Royle) Hulten. }\end{array}$ & $\mathrm{P}$ & $\mathrm{H}$ & $4000 \mathrm{~m}$ & $\mathrm{C}$ & Not known & LP \\
\hline 91 & Saxifragaceae & Saxifraga jacquemontiana Decne. & $\mathrm{P}$ & $\mathrm{H}$ & $4200 \mathrm{~m}$ & Inf. & Not known & LP \\
\hline 92 & Saxifragaceae & Saxifraga pulvinaria $\mathrm{H}$. Smith & $\mathrm{P}$ & $\mathrm{H}$ & $4200 \mathrm{~m}$ & Inf. & Not known & LP \\
\hline 93 & Saxifragaceae & Saxifraga sibirica $\mathrm{L}$. & A-B & Th & $3700 \mathrm{~m}$ & Inf. & Not known & LP \\
\hline 94 & Scrophulariaceae & Lagotis kunawurensis (Royle) Rupr. & $\mathrm{P}$ & $\mathrm{H}$ & $3800 \mathrm{~m}$ & $\mathrm{C}$ & Not known & LP \\
\hline 95 & Scrophulariaceae & Pedicularis albida Penn. & $\mathrm{P}$ & $\mathrm{H}$ & $3700 \mathrm{~m}$ & $\mathrm{C}$ & Not known & LP \\
\hline 96 & Scrophulariaceae & Pedicularis multiflora Pennell & $\mathrm{P}$ & $\mathrm{H}$ & $3600 \mathrm{~m}$ & $\mathrm{C}$ & Not known & LP \\
\hline 97 & Scrophulariaceae & Pedicularis pyramidata Royle & $\mathrm{P}$ & $\mathrm{H}$ & $3600 \mathrm{~m}$ & $\mathrm{C}$ & Not known & LP \\
\hline 98 & Scrophulariaceae & Pedicularis tenuirostris Benth. & $P$ & $\mathrm{H}$ & $3700 \mathrm{~m}$ & $\mathrm{C}$ & Not known & HP \\
\hline 99 & Scrophulariaceae & Veronica alpina $\mathrm{L}$. & $P$ & $\mathrm{H}$ & $4400 \mathrm{~m}$ & $\mathrm{C}$ & Not known & $\mathrm{HP}$ \\
\hline 100 & Scrophulariaceae & Veronica macrostemon Bunge ex Ledeb. & $\mathrm{P}$ & $\mathrm{H}$ & $4000 \mathrm{~m}$ & Inf. & Not known & HP \\
\hline 101 & Valerianaceae & Valeriana hardwickii Wall.var. hoffmeisteri (KI.) Clarke & A & Th & $4000 \mathrm{~m}$ & $\mathrm{C}$ & High medicinal value & LP \\
\hline 102 & Valerianaceae & Valeriana jaeschkei C.B.Clarke & $\mathrm{P}$ & $\mathrm{H}$ & $4000 \mathrm{~m}$ & $\mathrm{C}$ & High medicinal value & LP \\
\hline 103 & Valerianaceae & Valeriana pyrolifolia Decne. & A & $\mathrm{Th}$ & $4200 \mathrm{~m}$ & $\mathrm{C}$ & High medicinal value & LP \\
\hline
\end{tabular}

Abbriviation: $\mathrm{T}=$ Tree, $\mathrm{Sh}=$ Shrub, $\mathrm{P}=$ Perennial, $\mathrm{B}=$ Biennial, $\mathrm{A}=$ Annual, $\mathrm{Ph}=$ Phanaerophytes, $\mathrm{Ch}=\mathrm{Chamaephyte}, \mathrm{H}=\mathrm{Hemicryp}$ tophyte, Th =

Therophyte, $\mathrm{G}=$ Geophyte, $\mathrm{Hy}=$ Hydrophyte, $\mathrm{CC}=$ Very common, $\mathrm{C}=$ Common, Inf. $=$ Infrequent, $\mathrm{R}=\mathrm{Rare}, \mathrm{RR}=\mathrm{Very} \mathrm{Rare}, \mathrm{HP}=\mathrm{High}$ palatable, $\mathrm{LP}=$ low Palatable, $\mathrm{NP}=$ Non palatable

Table 2. The dominant families of exclusively alpine species and dominant exclusive alpine genera and the life-form categories in the alpine zone of both valleys.

\begin{tabular}{lllllll}
\hline Sr. & Families & No. of Spp. & Genera & No. of Spp. & Life-form & \% \\
\hline 1 & Asteraceae & 16 & Carex & 9 & Hemicryptophyte & $85(80.95 \%)$ \\
2 & Ranunculaceae & 11 & Draba & 8 & Therophyte & $5(4.76 \%)$ \\
3 & Rosaceae & 10 & Rhodiola & 5 & Phanaerophytes & $2(1.90 \%)$ \\
4 & Cyperaceae & 10 & Pleurospermum & 4 & Geophyte & $5(4.76 \%)$ \\
5 & Brassicaceae & 10 & Delphinium & 3 & Chamaephyte & $8(7.61 \%)$ \\
6 & Scrophulariaceae & 7 & Aconitum & 2 & \\
7 & Poaceae & 6 & Pseudomertensia & 2 & \\
8 & Crassulaceae & 5 & Gagea & 2 & \\
9 & Apiaceae & 5 & Festuca & & \\
10 & Saxifragaceae & 4 & & & \\
11 & Polygonaceae & 3 & & & \\
12 & Liliaceae & 3 & & & \\
13 & Valarianaceae & 3 & & & \\
\hline
\end{tabular}

(with 9 species each), followed by Draba (8 species) and Rhodiola (with 5 species) (Table 2). The flora of the both valleys has close affinities with the flora of Himalayas. The altitudinal distribution of exclusively alpine species in both valleys shows highest number of 80 species in the lower habitats from $3600-4000 \mathrm{~m}$ of alpine zone and 25 species were distributed in the high altitudes habitats more than $4000 \mathrm{~m}$. Out of 103 species identified 20 and more species were being used to cure different diseases. These 20 species have highly medicinally importance among the peoples of both valleys. 
The area is belongs to the Irano-Turanian region where the rate of endemism is generally high in upper areas, where 3 exclusively alpine endemic and 25 infrequent species were found, while the rate of endemism is low in lower areas of this zone. Rhododendron is the only species being widespread in north and west facing slopes of alpine zone. A very rare species Doronicum falconeri was also found in this zone. During last 13 years only few individuals of this species were seen in the alpine zone of Haramosh valley. Thus, the topography and micro-climate of a particular region may determine the vegetation pattern. In such cases, the herbaceous flora is a helpful aid in recognizing the alpine zone.

\section{DISCUSSION}

The present study was focused only on the alpine exclusive species and genera of Haramosh and Bagrote valleys of the Karakoram Range above tree line. During the study we identified 103 exclusively alpine species belongs to 52 genera and 21 families of Angiosperms. Among the 21 families, 4 families were represented by only one species each, 12 families had 2 to 7 species each, while only 5 families contained 10 or more species. Collectively these 5 larger families had 57 species (52.28\% of the total species) and 25 genera of Alpine flora belonged to these families. Like species genera were also found to exclusive in alpine zone. The study revealed that herbs appear to be dominant with 103 species i.e. $98.09 \%$; shrubs are represented by 2 species $(1.90 \%)$. The ratio of Monocots was found $20.95 \%$ while dicots $79.04 \%$ found at species level. This shows that the diversity of Dicots in comparison to that of monocots is much higher in the study area. The dominant species found in this region are those which can tolerate the grazing pressure and grow in dry habitats in open sunny slopes of alpine pastures. The widely distributed species of alpine zone are mostly belongs to the families like Asteraceae Rosaceae, Gentianaceae, Scrophulariaceae, Polygonaceae, Cyperaceae, Brassicaceae, and Poaceae, etc. Therefore, the present study has revealed that the area provides suitable habitat for the species of Potentilla, Bistorta, Pedicularis, Geranium, Gentianoides and Aster, etc. The alpine meadows of both valleys are dominated by members of family Cyperace, such as Carex divisa, Eleocharis quinqueflora, Kobresia laxa and Carex pseudofoetida in all the moist and open meadows of alpine zone up to snow belt. The Cyperaceae mats play a vital environmental role of protecting large alpine areas against erosion (Miehe et al., 2008). In the Alpine zone Asteraceae was floristically rich family containing 16 alpine exclusive species, followed by Ranunculaceae with 11 alpine specific species and Brassicaceae, Rosaceae and Cyperaceae were floristically the third large families with 10 species each. Like the larger families, the larger genera also showed a tendency to be more concentrated towards the higher altitudes. In the
Alpine zone, 37 species belonged to 9 of these alpine exclusive larger genera, constituting $35.23 \%$ of the total alpine exclusive species of this zone. Similarly, Hemicryptophytes were found to dominant in Alpine specific flora. The alpine zones are frequently rich in species of hemicryptopytes (Naroozi et al., 2008). The perennial herbs formed $93.33 \%$ of the Alpine flora while Anuual/Biennial herbs formed $4.76 \%$ and Phanerophytes were only $1.90 \%$ of the Alpine exclusive flora. In the Alpine zone, species of Potentilla, Carex, Eleochris etc. were dominant. It was observed that Potentilla species were not only grazingresistant but also efficient in reproduction, and so were the Bistorta species. Therefore, these species seem to increase in response to grazing pressure by the livestock. Due to rising temperature, the migration of species from Subalpine to Alpine zone has been recorded, while some Alpine species like Saussurea simpsoniana have now become restricted to the upper most part of their distributional ranges. It was observed that the species of lower margins such as Corydalis spp. Veronica alpina, Cremanthodium decaisnei etc. were migrating upwards and dislodging Saussurea simpsoniana from its habitat continuously. There is a proof of an ongoing upward migration, a decline of subnival-nival species at their lower range limits in the Alps (Pauli et al., 2007) and the lower elevations may support an upward migration motivated by climate change (Noroozi, et al. 2011). According to fourteen years observation Potentilla atrosanguinea, Potentilla anserina, Bistorta affinis, Aconogonon alpinum, Silene gonosperma, Papaver nudicaule, Anemone rupicola, Primula elliptica, Allium oreoprasum, Pulsatilla wallichiana, were found to upward shift from sub alpine to alpine zone. The accumulation of larger genera and zone-specific species into the Alpine zone had been due to their gradual migration from the lower altitudes over the decades. Such type of studies from the other parts of the world have documented or predicted the migration of species in response to global warming. Lenior et al. (2008) reported 171 forest species of W. Europe upward shifted an average 29 meter per decade from 1986 to 2005 . As observed in the present study, there is a high number of genera and species that are exclusively found in the Alpine zone of the study area. Climate change has strongly influenced distribution and abundance of plants at range margins both in latitude and elevation (Tryjanowski, 2005; Hickling et al., 2006). In the five years of monitoring, some species, particularly the dominant ones, continued to increase their IVI values, but several others decreased and some disappeared from their habitats. The alpine zone was dominated by Potentilla atrosanguinea, Potentilla anserina Carex divisa, Geranium pratense and Eleocharis quinqueflora. The first three species increased their IVI over the years, while Geranium pratense decreased and Eleocharis quinqueflora remained more or less same. Two other species Alchemilla ypsilotoma and Nepeta nervosa were not found during the first one and two years of monitoring, respectively. 
However, after their appearance they continued to increase their IVI values. Primula denticulata, Allardia glabra, Euphorbia micractina, Pyrola rotundifolia, Pusatilla wallichiana and Anemone rupicola showed sharp decrease, and the last two were not found in the fifth year of monitoring (Table 3). Loarie et al. (2008) suggest that climate change has the potential to break up local floras resulting in new species mixes, with consequent novel patterns of competition and other biotic interactions. Similarly, the distribution of larger genera (those with 5 or more species each) also showed greater concentration towards higher altitudes.

Table 3. Comparison of IVI values from 2003-2007 in Alpine zone of Haramosh and Bugrote Valley.

\begin{tabular}{|c|c|c|c|c|c|c|}
\hline \multirow[t]{2}{*}{ Sr. } & \multirow[t]{2}{*}{ Name of Species } & \multirow{2}{*}{$\begin{array}{c}2003 \\
\text { IVI } \\
\end{array}$} & \multirow{2}{*}{$\begin{array}{c}2004 \\
\text { IVI }\end{array}$} & \multirow{2}{*}{$\begin{array}{c}2005 \\
\text { IVI } \\
\end{array}$} & \multirow{2}{*}{$\begin{array}{c}2006 \\
\text { IVI }\end{array}$} & \multirow{2}{*}{$\begin{array}{c}2007 \\
\text { IVI }\end{array}$} \\
\hline & & & & & & \\
\hline 1 & Potentilla atrosanguinea & 19.41 & 21.45 & 23.60 & 26.67 & 28.59 \\
\hline 2 & Potentilla anserine & 21.14 & 22.80 & 24.12 & 24.63 & 24.64 \\
\hline 3 & Carex divisa & 13.18 & 13.29 & 14.53 & 16.32 & 20.01 \\
\hline 4 & Geranium pretense & 25.44 & 26.34 & 26.96 & 22.92 & 18.37 \\
\hline 5 & Eleocharis quinqueflora & 12.45 & 14.74 & 15.63 & 15.76 & 15.78 \\
\hline 6 & Bistorta affinis & 8.01 & 9.27 & 10.61 & 10.69 & 13.96 \\
\hline 7 & Kobresia laxa & 11.56 & 11.21 & 12.78 & 13.44 & 13.46 \\
\hline 8 & Oxytropis immerse & 9.32 & 8.90 & 9.43 & 10.47 & 10.85 \\
\hline 9 & Astragalus hendersonii & 8.98 & 8.55 & 9.05 & 9.29 & 10.62 \\
\hline 10 & Carex pseudofoetida & 10.04 & 9.24 & 8.32 & 8.48 & 9.75 \\
\hline 11 & Gentianodes tianschanica & 7.63 & 7.83 & 7.58 & 7.70 & 8.95 \\
\hline 12 & Alchemilla ypsilotoma & 0.00 & 4.09 & 2.17 & 4.01 & 8.95 \\
\hline 13 & Poa alpine & 7.81 & 7.38 & 7.37 & 7.92 & 8.33 \\
\hline 14 & Aconogonon alpinum & 6.89 & 6.34 & 5.98 & 10.15 & 8.11 \\
\hline 15 & Poa stapfiana & 5.50 & 5.78 & 6.12 & 7.04 & 7.40 \\
\hline 16 & Elymus dahuricus & 7.19 & 6.72 & 7.126 & 6.13 & 6.53 \\
\hline 17 & Festuca alaica & 7.03 & 5.95 & 5.25 & 5.28 & 6.50 \\
\hline 18 & Nepeta nervosa & 0.00 & 0.00 & 2.32 & 2.84 & 6.33 \\
\hline 19 & Swertia petiolate & 6.73 & 8.35 & 6.89 & 5.87 & 6.23 \\
\hline 20 & Erigeron alpinum & 5.44 & 4.53 & 4.80 & 5.68 & 6.03 \\
\hline 21 & Silene gonosperma & 5.07 & 4.56 & 4.83 & 4.83 & 5.60 \\
\hline 22 & Aster peduncularis & 8.07 & 7.22 & 5.90 & 5.01 & 5.41 \\
\hline 23 & Rhodiola wallichiana & 5.55 & 6.72 & 7.11 & 7.08 & 4.35 \\
\hline 24 & Sibbaldia procumbens & 3.87 & 3.77 & 3.54 & 3.54 & 3.93 \\
\hline 25 & Senecio tibeticus & 6.43 & 3.70 & 3.92 & 3.91 & 3.86 \\
\hline 26 & Rhodiola heterodonta & 4.85 & 5.15 & 5.46 & 5.43 & 3.86 \\
\hline 27 & Aster falconeri & 5.97 & 5.46 & 5.23 & 3.84 & 3.39 \\
\hline 28 & Gentianodes eumarginata & 6.11 & 7.29 & 6.37 & 7.30 & 3.35 \\
\hline 29 & Phleum alpinum & 4.97 & 4.05 & 3.93 & 3.90 & 3.27 \\
\hline 30 & Pedicularis staintonii & 4.26 & 0.00 & 3.10 & 2.64 & 3.04 \\
\hline 31 & Primula denticulate & 7.58 & 7.11 & 7.08 & 0.00 & 3.01 \\
\hline 32 & Tanacetum falconeri & 3.09 & 3.75 & 3.98 & 3.90 & 2.99 \\
\hline 33 & Papaver nudicaule & 3.30 & 2.68 & 2.84 & 0.00 & 2.81 \\
\hline 34 & Valeriana himalayana & 4.57 & 5.22 & 5.33 & 2.98 & 2.68 \\
\hline 35 & Allardia glabra & 4.49 & 3.50 & 3.71 & 3.24 & 2.51 \\
\hline 36 & Chorispora macropoda & 3.16 & 2.93 & 2.47 & 2.46 & 2.21 \\
\hline 37 & Euphorbia micractina & 3.77 & 3.58 & 0.00 & 2.18 & 2.18 \\
\hline 38 & Pedicularis albida & 3.61 & 3.85 & 4.08 & 4.05 & 2.00 \\
\hline 39 & Pyrola rotundifolia & 3.11 & 3.34 & 0.00 & 1.30 & 1.26 \\
\hline 40 & Pulsatilla wallichiana & 9.54 & 8.22 & 6.97 & 6.69 & 0.00 \\
\hline 41 & Anemone rupicola & 4.68 & 4.94 & 3.34 & 4.22 & $0.00 *$ \\
\hline
\end{tabular}

*Abbreviation: Important value index (IVI); Zero in the IVI column shows absence

The current study revealed that in the Alpine zone, 105 exclusive alpine species belonged to 52 genera, which constituted $45.6 \%$ of total 232 species of Alpine zone. The alpine slopes provide an array of microclimates with differences in temperature, precipitation and solar radiation. Giriraj et al. (2008) demonstrated the distribution of species assemblages along an altitudinal gradient, and the occurrence of certain species unique to a particular altitude or habitat. The two valleys, Haramosh and Bagrote had quite similar flora with a high value of Similarity Index. However, the alpine zone of Haramosh valley was floristically richer than the Bagrote valley. The up-to-date information on biodiversity is critical for the proper management and conservation of any area, thus the first step towards conservation should be to compile a species inventory or checklist (Droege and Larivee, 1998; Salafsky et al., 2002). The present study is the first detailed work on the alpine specific studies regarding, alpine exclusive species and genera, their distribution, and conservation which may helpful in such studies in any part of the Northern Areas of Pakistan, involving thorough inventorying and monitoring of plant biodiversity.

\section{REFERENCES}

Ali, S. I. and M. Qaiser (Eds.). 1993-2012. Flora of PakistanEd. Nos. 194-215. Department of Botany University of Karachi and National Herbarium Islamabad, Pakistan.

Ali, S.I. and M. Qaiser. 1986. A phytogeographic analysis of the phanerogams of Pakistan and Kashmir. Proc. of the Royal Soc. Edinburgh. 89B, 89 -101.

Ali, S.I. and Y.J. Nasir (Eds.). 1989-1991. Flora of PakistanNos. 191-193. Department of Botany, University of Karachi and National Herbarium Islamabad, Pakistan.

Dodd, J.L. 1994. Desertification and degradation in subSaharan Africa. Biosci. 44:28-34.

Droege, S., A. Cyr and J. Larivee. 1998. Checklists: an under used tool for the inventorying and monitoring of plants and animal. Conserv. Biol. 12:1134-1138.

Dugan, L.E., M.F. Wojciechowski and L.R. Landrum. 2007. A large scale plant survey: efficient vouchering with identification through morphology and DNA analysis. Taxon. 56:1238-1244.

Ellenberg, H., H.E. Weber, R. Dull, V. Wirth, W. Werner and D. Pauliben. 1991. Zeigwerte von Pflanzen in Mitteleuropa. Scripta. Geo. Bot. 18:1-248.

Giriraj, A., M.S.R. Murthy and B.R. Ramesh. 2008. Vegetation composition, structure, and patterns of diversity: A case study from the tropical wet evergreen forest of the Western Ghats, India. Edinburgh J. Bot. 65:447-468.

Hardy, J.T. 2003. Climate Change: Causes, Effects and Solutions. John Wiley and Sons Ltd., England; p.40.

Hickling, R., D.B. Roy, K.J. Hill, R. Fox and C.D. Thomas. 2006. The distributions of a wide range of taxonomic groups are expanding polewards. Glob. Change Biol. $12: 450-455$. 
Khan, S.W. and S. Khatoon. 2007. Ethnobotanical studies on useful trees and shrubs of Haramosh and Bugrote valleys in Gilgit Northern Areas of Pakistan. Pak. J. Bot. 39:699710.

Kumar, S. and S. Sharma. 2013. Species diversity, uses and distribution of medicinal plants along an altitudinal gradient in North Western Himalayas. Int. J. Med. Arom. Plants V. 3:343-351.

Lenoir, J., J.C. Gegout, P.A. Marquet, P. DeRuffray and H. Brisse. 2008. A significant upward shift in plant species optimum elevation during the $20^{\text {th }}$ Century. Sci. 320:1768-1771.

Loarie, S.R., B.E. Carter and K. Hayhoe. 2008. Climate change and the future of California's endemic flora. PLOS ONE 3(6):e2502.

Miehe, G., S. Miehe, K. Kaiser, L. Jianquan and X. Zhao. 2008. Status and dynamics of the Kobresia pygmaea ecosystem on the Tibetan plateau. Ambio. 37:272-279.

Nasir, E. and S.I. Ali (Eds.). 1970-1989. Flora of PakistanNos. I-190. Department of Botany University of Karachi and National Herbarium, Islamabad, Pakistan.

Noroozi, J., H. Akhani and S.W. Breckle. 2008. Biodiversity and phytogeography of the alpine flora of Iran. Biod. Conserv. 17:493-521.

Noroozi, J., H. Pauli, G. Grabherr and S.W. Breckle. 2011. The subnival-nival vascular plant species of Iran: a unique high-mountain flora and its threat from climate warming. Biod. Conserv. 20:1319-1338.

Pauli, H., M. Gottfried and K. Reiter. 2007. Signals of range expansions and contractions of vascular plants in the high Alps: observations (1994-2004) at the GLORIA master site Schrankogel, Tyrol, Austria. Glob. Change Biol. 13:147-156.

Perkin, S. 2003. Northern Areas- State of Environment and Development. Government of Pakistan and IUCN Karachi, Pakistan.

Ramanathan, V. 2007. Warming trends in Asia amplified by brown cloud solar absorption. Nature 448:575-578.

Raunkiaer, C. 1934. Life-Forms of Plants and Statistical Plant Geography. Clarendon Press, Oxford.

Ronell, R.K., L. Gautier, C. Chatelain, G.F. Smith and R. Spichiger. 2007. Floristics of the angiosperms flora of Sub-Saharan Africa: an analysis of the African plant checklist and database. Taxon. 56:201-208.

Salafsky, N., R, Margoluis, K.H. Redford and J.G. Robinson. 2002. Improving the practice of conservation: a conceptual framework and research agenda for conservation science. Conserv. Biol. 16:1469-1479.

Stewart, R.R. 1972. An annotated catalogue of the vascular plants of Western Pakistan and Kashmir. Department of Botany, University of Karachi, Karachi-Pakistan.

Stork, N.E. and M.J. Samways. 1995. Inventorying and monitoring. In: V.H. Heywood (ed.), Global Biodiversity Assessment. Cambridge University Press Cambridge; pp.459-543.

Tryjanowski, P., T.H. Sparks and P. Profus. 2005. Uphill shifts in the distribution of the white stork Ciconia. Diversity Distrib. 11:219-223.

Watson, I. and P. Novelly. 2004. Making the biodiversity monitoring system sustainable: Design issues for large scale monitoring system. Aust. Eco. 29:16-30. 